\title{
Darboux transformation, bright and dark-bright solitons of an $N$-coupled high-order nonlinear Schrödinger system in an optical fiber
}

Xi-Hu Wu, Yi-Tian Gao, Xin Yu† Cui-Cui Ding, Fei-Yan Liu, Ting-Ting Jia

\author{
Ministry-of-Education Key Laboratory of Fluid Mechanics and National \\ Laboratory for Computational Fluid Dynamics, Beijing University of \\ Aeronautics and Astronautics, Beijing 100191, China
}

\begin{abstract}
In this paper, an $N$-coupled high-order nonlinear Schrödinger system, which describes the properties of the ultrashort optical pulses in an optical fiber, is investigated with the help of Darboux transformation (DT) method and asymptotic analysis. Starting from the given $(2 N+1)$ th-order Lax pair, we construct a new form of DT (complex eigenfunctions of Lax pair involved) to derive the formulas of the $n$ th-iterated solutions, where $n$ and $N$ are the positive integers. On the zero background, the first- and second-order solitons are obtained and analysed through the asymptotic analysis. Multi-parameter adjustment is proceeded since there are $3 N+4$ real parameters in the second-order solitons. We find that under certain conditions each of the two interaction patterns (elastic, inelastic) holds in the second-order soliton. On the plane wave background, the first-order bright and dark-bright solitons are obtained. Soliton velocities, amplitudes, widths and characteristic lines of the first-order bright and dark-bright solitons are presented and analysed.
\end{abstract}

Keywords: Optical fiber; $N$-coupled high-order nonlinear Schrödinger system; Darboux transformation; Dark-bright solitons; Soliton interaction; Asymptotic analysis

${ }^{*}$ Corresponding author, with e-mail address as gaoyt2021@163.com

${ }^{\dagger}$ Corresponding author, with e-mail address as yuxin@buaa.edu.cn 


\section{Introduction}

Nonlinear evolution equations have been used to describe the physical phenomena in fluid mechanics, fiber optics, biochemistries and plasma physics [1-7]. Solitons, as a kind of solution of the nonlinear evolution equations, have attracted the attention of the researchers [8, 9].

An $N$-coupled high-order nonlinear Schrödinger (HNLS) system has been proposed to describe the properties of the ultrashort optical pulses in an optical fiber [10-15]:

$$
i u_{j Z}+\frac{1}{2} u_{j T T}+u_{j} \sum_{n=1}^{N}\left|u_{n}\right|^{2}+i\left[u_{j T T T}+6 u_{j T} \sum_{n=1}^{N}\left|u_{n}\right|^{2}+3 u_{j}\left(\sum_{n=1}^{N}\left|u_{n}\right|^{2}\right)_{T}\right]=0(j=1,2, \ldots, N),
$$

where $u_{j}=u_{j}(Z, T)$ denotes the amplitude of the $j$ th component of a slowly varying field, $i=\sqrt{-1}, n$ and $N$ are the positive integers, the subscripts $Z$ and $T$ denote the normalized space and time derivatives, respectively. $u_{j T T T}$ represents the third-order dispersion, $6 u_{j T} \sum_{n=1}^{N}\left|u_{n}\right|^{2}$ represents the self-steepening and $3 u_{j}\left(\sum_{n=1}^{N}\left|u_{n}\right|^{2}\right)_{T}$ represents the stimulated Raman scattering effects [11].

For System (1), Hirota bilinear form [10], bright and dark solitons through the Hirota method [11], infinitely-many conservation laws [12], inverse scattering transformation [12], Painlevé singularity structure [13], Darboux transformation (DT) involving certain real eigenfunctions [13], mixed-type one- and two-soliton through the Hirota method [14] and Darboux-Bäcklund transformation [15] have been studied.

Via the variable transformations $[15,16]$

$$
\begin{aligned}
& q_{j}(x, t)=u_{j}(Z, T) e^{-\frac{i}{6}\left(T-\frac{Z}{18}\right)}, \\
& t=Z, \quad x=T-\frac{Z}{12},
\end{aligned}
$$

System (1) has been reduced to the $N$-coupled complex modified Korteweg-de Vries-type system of the form $[17,18]$ :

$$
q_{j t}+q_{j x x x}+6 q_{j x} \sum_{n=1}^{N}\left|q_{n}\right|^{2}+3 q_{j}\left(\sum_{n=1}^{N}\left|q_{n}\right|^{2}\right)_{x}=0
$$

For System (3), Riemann-Hilbert problem [17] and Hirota bilinear technique [18] have been studied.

Special cases of System (1) have been seen as follows:

- When $N=1$, System (1) has been reduced to the Sasa-Satsuma equation whose Bäcklund transformation [19], infinitely-many conservation laws [19] and Darboux dressing transformation [20] have been investigated. Sasa-Satsuma equation has been used to describe the development of the complex scalar field and evolutions of the short optical pulses [19]. 
- When $N=2$, System (1) has been reduced to the two-coupled HNLS equations to describe the pulses propagation in the coupled fibers [21]. Its Painlevé singularity structure [21], DT [22], infinitely-many conservation laws [23] and Darboux-Bäcklund transformation [24] have been investigated.

However, to our knowledge, for System (1), a DT involving certain complex eigenfunctions, which is different from DT (20) in Ref. [13], has not been studied. Meanwhile, two solitons on the zero background and solitons on the plane wave background have not been investigated. In Sect. 2, such a DT of System (1) will be constructed, and the $n$ th-iterated solutions of System (1) will be derived through that DT. In Sect. 3, through that DT and certain zero seed solutions, the first- and second-order soliton of System (1) will be derived on the zero background, and asymptotic analysis will be used to discuss the interaction between the two solitons. In Sect. 4, the first-order bright and dark-bright soliton of System (1) will be obtained on the plane wave background. In Sect. 5, the conclusions will be drawn.

\section{DT of System (1)}

Since System (1) and System (3) can be converted to each other through Variable Transformation (2), we can get the solutions of System (3) first, then perform Variable Transformation (2) and get the solutions of System (1).

Lax pair of System (3) has been obtained as [17]:

$$
\left\{\begin{array}{l}
\Phi_{x}=U \Phi, U=U_{1} \lambda+U_{0} \\
\Phi_{t}=V \Phi, V=V_{3} \lambda^{3}+V_{2} \lambda^{2}+V_{1} \lambda+V_{0}
\end{array}\right.
$$

where $U_{1}=i \sigma_{3}, U_{0}=A, V_{3}=4 i \sigma_{3}, V_{2}=4 A, V_{1}=2 i\left(A^{2}+A_{x}\right) \sigma_{3}, V_{0}=A_{x} A-A A_{x}-A_{x x}+2 A^{3}$, $\sigma_{3}=\operatorname{diag}(-1,1, \ldots, 1,1)$ and

$$
A=\left(\begin{array}{cccccc}
0 & r_{1} & s_{1} & \ldots & r_{N} & s_{N} \\
-s_{1} & 0 & 0 & \ldots & 0 & 0 \\
-r_{1} & 0 & 0 & \ldots & 0 & 0 \\
\ldots & \ldots & \ldots & \ldots & \ldots & \ldots \\
-s_{N} & 0 & 0 & \ldots & 0 & 0 \\
-r_{N} & 0 & 0 & \ldots & 0 & 0
\end{array}\right)
$$

with $q_{j}=r_{j}, q_{j}^{*}=s_{j}$ (the asterisk denotes the complex conjugation) and $\Phi=\Phi(x, t ; \lambda)$ is a $(2 N+1)$-dimensional column vector eigenfunction corresponding to the complex spectral parameter $\lambda$. System (3) can be obtained from the zero curvature equation $U_{t}-V_{x}+U V-V U=0$ of Lax Pair (4).

Motivated by Ref. [25], a DT is constructed as

$$
\Phi^{[1]}=T^{[1]} \Phi=\left(\lambda I-W^{[1]}\right) \Phi
$$


where $I$ is the $(2 N+1)$ th-order identity matrix, $W^{[1]}$ is a $(2 N+1)$ th-order square matrix, $\Phi$ satisfies Lax Pair (4) for the initial potential $\left(q_{1}, q_{2}, \ldots, q_{N}\right)^{T}$ (the superscript $T$ denotes the conjugate transpose), $\Phi^{[1]}$ satisfies Lax pair (4) for the new potential $\left(q_{1}^{[1]}, q_{2}^{[1]}, \ldots, q_{N}^{[1]}\right)^{T}$ and the superscript $[n]$ denotes the $n$th iteration.

Through DT (5), Lax Pair (4) is transformed into a new one possessing the same matrix form as followed:

$$
\begin{aligned}
& \Phi_{x}^{[1]}=U^{[1]} \Phi^{[1]}, U^{[1]}=U_{1}^{[1]} \lambda+U_{0}^{[1]}, \\
& \Phi_{t}^{[1]}=V^{[1]} \Phi^{[1]}, V^{[1]}=V_{3}^{[1]} \lambda^{3}+V_{2}^{[1]} \lambda^{2}+V_{1}^{[1]} \lambda+V_{0}^{[1]},
\end{aligned}
$$

where $U^{[1]}, V^{[1]}$ have the same forms with $U, V$, respectively, except that the $r_{j}, s_{j}$ in the matrices $U, V$ are replaced with $r_{j}^{[1]}, s_{j}^{[1]}$ in the matrices $U^{[1]}, V^{[1]}$.

Combining Eqs. (4), (5) and (6), we get

$$
\begin{aligned}
& U^{[1]} T^{[1]}=T_{x}^{[1]}+T^{[1]} U, \\
& V^{[1]} T^{[1]}=T_{t}^{[1]}+T^{[1]} V .
\end{aligned}
$$

We expand Eqs. (7) and let the coefficients of each subterm of $\lambda$ be zero, then we get

$$
\begin{aligned}
& U_{1}^{[1]}=U_{1}, \\
& U_{0}-U_{0}^{[1]}+U_{1}^{[1]} W^{[1]}-W^{[1]} U_{1}=0, \\
& W_{x}^{[1]}+W^{[1]} U_{1}-U_{1}^{[1]} W^{[1]}=0, \\
& V_{3}^{[1]}=V_{3}, \\
& V_{2}-V_{2}^{[1]}+V_{3}^{[1]} W^{[1]}-W^{[1]} V_{3}=0, \\
& V_{1}-V_{1}^{[1]}+V_{2}^{[1]} W^{[1]}-W^{[1]} V_{2}=0, \\
& V_{0}-V_{0}^{[1]}+V_{1}^{[1]} W^{[1]}-W^{[1]} V_{1}=0, \\
& -W_{t}^{[1]}+V_{0}^{[1]} W^{[1]}-W^{[1]} V_{0}=0 .
\end{aligned}
$$

Eqs. (8a) and (8d) are satisfied automatically, while Eqs. (8b) and (8e) are satisfied if and only if

$$
\begin{aligned}
& w_{1,2 j}^{[1]}=\frac{r_{j}-r_{j}^{[1]}}{2 i}, \\
& w_{1,2 j}^{[1]}=w_{2 j+1,1}^{[1]}=-w_{1,2 j+1}^{[1] *}=-w_{2 j, 1}^{[1] *} .
\end{aligned}
$$

We set $\Phi=\left(\phi_{1}, \phi_{2}, \phi_{2}^{*}, \ldots, \phi_{N+1}, \phi_{N+1}^{*}\right)^{T}$, where $\phi_{h}(h=1,2, \ldots, N+1)$ is the function of $x$ and $t$. If $\Phi$ is a eigenfunction of Lax Pair (4) with $\lambda=\lambda_{1}$, then $\left(\phi_{2}^{*},-\phi_{1}^{*}, \ldots, 0\right)^{T}$, $\left(\phi_{2}, \quad 0, \quad-\phi_{1}^{*}, \ldots, 0\right)^{T}, \ldots,\left(\phi_{N+1}^{*}, 0, \ldots,-\phi_{1}^{*}, 0\right)^{T}$ and $\left(\phi_{N+1}, 0, \ldots,-\phi_{1}^{*}\right)^{T}$ are other eigenfunctions of Lax Pair (4) with $\lambda=\lambda_{1}^{*}$. We choose

$$
W^{[1]}=H^{[1]} J^{[1]}\left(H^{[1]}\right)^{-1},
$$


with

$$
\begin{gathered}
J^{[1]}=\left(\begin{array}{ccccc}
\lambda_{1} & 0 & 0 & \ldots & 0 \\
0 & \lambda_{1}^{*} & 0 & \ldots & 0 \\
0 & 0 & \lambda_{1}^{*} & \ldots & 0 \\
\vdots & \vdots & \vdots & \ddots & \vdots \\
0 & 0 & 0 & \ldots & \lambda_{1}^{*}
\end{array}\right), \\
H^{[1]}=\left(\begin{array}{cccccc}
\phi_{1}\left(\lambda_{1}\right) & \phi_{2}^{*}\left(\lambda_{1}\right) & \phi_{2}\left(\lambda_{1}\right) & \ldots & \phi_{N+1}^{*}\left(\lambda_{1}\right) & \phi_{N+1}\left(\lambda_{1}\right) \\
\phi_{2}\left(\lambda_{1}\right) & -\phi_{1}^{*}\left(\lambda_{1}\right) & 0 & \ldots & 0 & 0 \\
\phi_{2}^{*}\left(\lambda_{1}\right) & 0 & -\phi_{1}^{*}\left(\lambda_{1}\right) & \ldots & 0 & 0 \\
\vdots & \vdots & \vdots & \ddots & \vdots & \vdots \\
\phi_{N+1}\left(\lambda_{1}\right) & 0 & 0 & \ldots & -\phi_{1}^{*}\left(\lambda_{1}\right) & 0 \\
\phi_{N+1}^{*}\left(\lambda_{1}\right) & 0 & 0 & \ldots & 0 & -\phi_{1}^{*}\left(\lambda_{1}\right)
\end{array}\right)
\end{gathered}
$$

where $\left(H^{[1]}\right)^{-1}$ is the inverse of $H^{[1]}$. Different from Ref. [13], we only require that the first eigenfunction $\phi_{1}\left(\lambda_{1}\right)$ is real but the other eigenfunctions can be complex.

After $W^{[1]}$ is confirmed, Eq. (9a) is satisfied, but Eq. (9b) demands that $\operatorname{Re}\left(\lambda_{1}\right)=0$ ("Re" denotes the real part of a complex number) and $\phi_{1}\left(\lambda_{1}\right)$ is real. It can be proved that Eqs. (8c), (8f), (8g) and (8h) are valid.

Eq. (9a) gives the relations between $\left(q_{1}, q_{2}, \ldots, q_{N}\right)^{T}$ and $\left(q_{1}^{[1]}, q_{2}^{[1]}, \ldots, q_{N}^{[1]}\right)^{T}$ as followed:

$$
\begin{aligned}
q_{j}^{[1]} & =q_{j}-2 i w_{1,2 j}^{[1]} \\
& =q_{j}-2 i\left(\lambda_{1}-\lambda_{1}^{*}\right) \frac{\phi_{1}\left(\lambda_{1}\right) \phi_{j+1}^{*}\left(\lambda_{1}\right)}{\left|\phi_{1}\left(\lambda_{1}\right)\right|^{2}+2\left|\phi_{2}\left(\lambda_{1}\right)\right|^{2}+2\left|\phi_{3}\left(\lambda_{1}\right)\right|^{2}+\ldots+2\left|\phi_{N+1}\left(\lambda_{1}\right)\right|^{2}} .
\end{aligned}
$$

Now we have already constructed a DT of System (3), which involves complex eigenfunctions of the $(2 N+1)$ th-order Lax Pair (4). Eq. (13) gives the relation between the initial and new solutions of System (3). Eq. (5) gives the relation between the initial eigenfunction $\Phi$ and new eigenfunction $\Phi^{[1]}$ of Lax Pair (4).

Iterating the above steps $n$ times, the $n$ th-iterated solutions of System (3) are presented as:

$$
\begin{aligned}
& q_{j}^{[n]}=q_{j}-\sum_{k=1}^{n} 2 i\left(\lambda_{k}-\lambda_{k}^{*}\right) \frac{\phi_{1}^{[k]}\left(\lambda_{k}\right) \phi_{j+1}^{[k] *}\left(\lambda_{k}\right)}{\left|\phi_{1}^{[k]}\left(\lambda_{k}\right)\right|^{2}+2\left|\phi_{2}^{[k]}\left(\lambda_{k}\right)\right|^{2}+2\left|\phi_{3}^{[k]}\left(\lambda_{k}\right)\right|^{2}+\ldots+2\left|\phi_{N+1}^{[k]}\left(\lambda_{k}\right)\right|^{2}} \\
& \Phi^{[k]}\left(\lambda_{k}\right)=\left(\phi_{1}^{[k]}\left(\lambda_{k}\right), \phi_{2}^{[k]}\left(\lambda_{k}\right), \phi_{2}^{[k]}\left(\lambda_{k}\right), \ldots, \phi_{N+1}^{[k]}\left(\lambda_{k}\right), \phi_{N+1}^{[k]}\left(\lambda_{k}\right)\right)^{T} \\
&=\left(\lambda_{k} I-W^{[k-1]}\right)\left(\lambda_{k} I-W^{[k-2]}\right) \ldots\left(\lambda_{k} I-W^{[1]}\right) \Phi\left(\lambda_{k}\right),
\end{aligned}
$$

where $k=1,2, \ldots, n, \Phi^{[k]}\left(\lambda_{k}\right)$ is the eigenfunction of Lax Pair (4) with $\left(q_{1}^{[k-1]}, q_{2}^{[k-1]}, \ldots, q_{N}^{[k-1]}\right)$ and $\lambda=\lambda_{k}$.

Via Variable Transformation (2), we can get the $n$ th-iterated solutions of System (1)

$$
u_{j}^{[n]}[Z, T]=q_{j}^{[n]}[Z, T] e^{\frac{i}{6}\left(T-\frac{Z}{18}\right)},
$$


where $q_{j}^{[n]}[Z, T]$ is converted from $q_{j}^{[n]}[x, t]$.

Eqs. (14)-(16) infer that we can obtain the $n$ th-iterated solutions of System (1) with the initial seed solutions. There are three points that need to be explained: (i) The whole iterative process does not contain differential and integral operations. We only need to solve the linear Lax Pair (4) and obtain new solutions through repetitive matrix operations. (ii) The expressions of the $n$ th-iterated solutions contain certain free parameters, e.g., spectral parameter $\lambda_{k}$ and integral constants in the seed solutions. (iii) DT (5) demands that $\lambda_{k}$ must be a pure imaginary number and $\phi_{1}\left(\lambda_{k}\right)$ must be real.

\section{Bright solitons of System (1) on the zero background}

\subsection{The first-order soliton of System (1)}

In order to derive solitons of System (3), we take seed solutions as $q_{1}=q_{2}=\ldots=q_{N}=0$ and let $\lambda_{1}=i \eta_{1}\left(\eta_{1} \in R\right.$ and $\left.\eta_{1} \neq 0\right)$. Substituting the seed solutions into Lax Pair (4), we can get eigenfunctions as followed:

$$
\begin{aligned}
& \phi_{1}\left(\lambda_{1}\right)=e^{\eta_{1} x-4 \eta_{1}^{3} t+\delta_{1}}, \\
& \phi_{2}\left(\lambda_{1}\right)=e^{-\eta_{1} x+4 \eta_{1}^{3} t+\delta_{2 R}+i \delta_{2 C}}, \\
& \cdots \\
& \phi_{N+1}\left(\lambda_{1}\right)=e^{-\eta_{1} x+4 \eta_{1}^{3} t+\delta_{N+1, R}+i \delta_{N+1, C}},
\end{aligned}
$$

where $\delta_{1}, \delta_{2 R}, \delta_{3 R}, \ldots, \delta_{N+1, R}, \delta_{2 C}, \delta_{3 C}, \ldots, \delta_{N+1, C}$ are all real constants, the subscript $R$ denotes the real part and the subscript $C$ denotes the imaginary part.

Taking the Eigenfunctions (17) into Eq. (13), we can get the first-order soliton of System (3)

$$
\begin{aligned}
& q_{j}^{[1]}=2 \eta_{1} e^{\delta_{j+1, R}-i \delta_{j+1, C}-\delta} \operatorname{sech}\left(8 \eta_{1}^{3} t-2 \eta_{1} x-\delta_{1}+\delta\right), \\
& \delta=\frac{1}{2} \ln \left[2\left(e^{2 \delta_{2 R}}+\ldots+e^{2 \delta_{N+1, R}}\right)\right] .
\end{aligned}
$$

Via Variable Transformation (2), we can get the first-order soliton of System (1)

$$
u_{j}^{[1]}=2 \eta_{1} e^{\delta_{j+1, R}-i\left[\delta_{j+1, C}+\frac{1}{6}\left(\frac{Z}{18}-T\right)\right]-\delta} \operatorname{sech}\left[\left(8 \eta_{1}^{3}+\frac{1}{6} \eta_{1}\right) Z-2 \eta_{1} T-\delta_{1}+\delta\right] .
$$

From Solutions (19), we can obtain the following soliton properties:

(a) Soliton Velocities: $V\left(u_{j}^{[1]}\right)=\frac{12}{48 \eta_{1}^{2}+1}$,

(b) Soliton Amplitudes: $A\left(u_{j}^{[1]}\right)=2\left|\eta_{1}\right| e^{\delta_{j+1, R}-\delta}$,

(c) Soliton Widths: $W\left(u_{j}^{[1]}\right)=\frac{1}{2 \eta_{1}}$,

(d) Total Soliton Energy: $E=\int_{-\infty}^{+\infty}\left(\left|u_{1}^{[1]}\right|^{2}+\ldots+\left|u_{N}^{[1]}\right|^{2}\right) \mathrm{d} x=4 \eta_{1}^{2}\left[\sum_{j=1}^{N} e^{2\left(\delta_{j+1, R}-\delta\right)}\right]$,

(e) Characteristic Lines: $\left(8 \eta_{1}^{3}+\frac{1}{6} \eta_{1}\right) Z-2 \eta_{1} T-\delta_{1}+\delta=0$.

We find that all the one solitons enjoy the same velocity, width and characteristic line. The soliton width is in inverse proportion to $\eta_{1}$ while the velocity decreases as $\left|\eta_{1}\right|$ increases, and the 
velocity is always positive. Different one solitons have different amplitudes due to the different $\delta_{j+1, R}$. The total energy keeps constant and it's independent with $\delta_{1}$. The first-order soliton for System (1) with $N=3$ is plotted in Figs. 1 in which the surface and shape of the one soliton are presented.

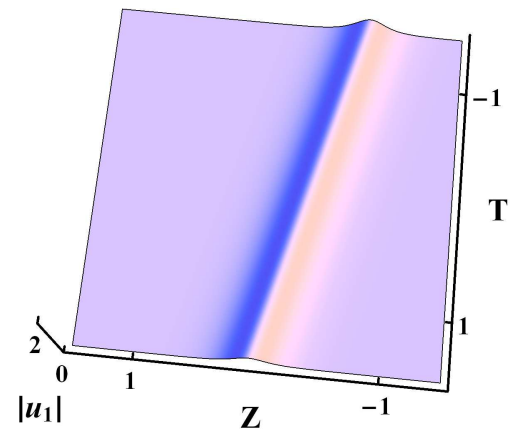

(a)

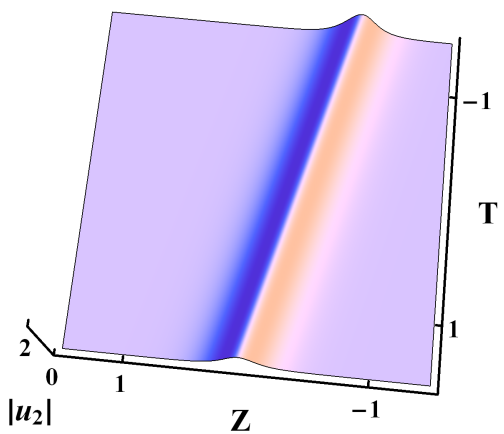

(b)

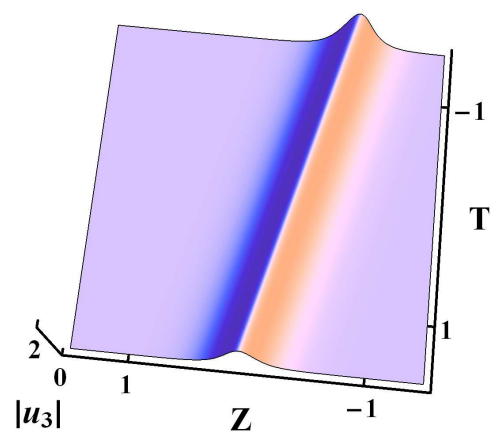

(c)

Figs. 1. One soliton via Solutions (19) with $N=3, \eta_{1}=1, \delta_{1}=2, \delta_{2 R}=3, \delta_{3 R}=3.5, \delta_{4 R}=4, \delta_{2 C}=1, \delta_{3 C}=1$ and $\delta_{4 C}=1$.

\subsection{The second-order soliton of System (1)}

We let $\lambda_{1}=i \eta_{1}$ and $\lambda_{2}=i \eta_{2}\left(\eta_{2} \in R\right.$ and $\left.\eta_{2} \neq 0\right)$, then the corresponding eigenfunctions are

$$
\begin{aligned}
& \phi_{1}\left(\lambda_{1}\right)=e^{\eta_{1} x-4 \eta_{1}^{3} t+\delta_{1}}, \\
& \phi_{2}\left(\lambda_{1}\right)=e^{-\eta_{1} x+4 \eta_{1}^{3} t+\delta_{2 R}+i \delta_{2 C}}, \\
& \ldots \\
& \phi_{N+1}\left(\lambda_{1}\right)=e^{-\eta_{1} x+4 \eta_{1}^{3} t+\delta_{N+1, R}+i \delta_{N+1, C}}, \\
& \phi_{1}\left(\lambda_{2}\right)=i e^{\eta_{2} x-4 \eta_{2}^{3} t+\xi_{1}}, \\
& \phi_{2}\left(\lambda_{2}\right)=i e^{-\eta_{2} x+4 \eta_{2}^{3} t+\xi_{2 R}+i \xi_{2 C}}, \\
& \ldots \\
& \phi_{N+1}\left(\lambda_{2}\right)=i e^{-\eta_{2} x+4 \eta_{2}^{3} t+\xi_{N+1, R}+i \xi_{N+1, C}},
\end{aligned}
$$

where $\xi_{1}, \xi_{2 R}, \xi_{3 R}, \ldots, \xi_{N+1, R}, \xi_{2 C}, \xi_{3 C}, \ldots, \xi_{N+1, C}$ are all real constants. From the $n$ th-iterated Solutions (14) with $n=2$, the second-order soliton is obtained as

$$
\begin{aligned}
q_{j}^{[2]}= & q_{j}-2 i\left(\lambda_{1}-\lambda_{1}^{*}\right) \frac{\phi_{1}\left(\lambda_{1}\right) \phi_{j+1}^{*}\left(\lambda_{1}\right)}{\left|\phi_{1}\left(\lambda_{1}\right)\right|^{2}+2\left|\phi_{2}\left(\lambda_{1}\right)\right|^{2}+2\left|\phi_{3}\left(\lambda_{1}\right)\right|^{2}+\ldots+2\left|\phi_{N+1}\left(\lambda_{1}\right)\right|^{2}} \\
& -2 i\left(\lambda_{2}-\lambda_{2}^{*}\right) \frac{\phi_{1}^{[1]}\left(\lambda_{2}\right) \phi_{j+1}^{[1] *}\left(\lambda_{2}\right)}{\left|\phi_{1}^{[1]}\left(\lambda_{2}\right)\right|^{2}+2\left|\phi_{2}^{[1]}\left(\lambda_{2}\right)\right|^{2}+2\left|\phi_{3}^{[1]}\left(\lambda_{2}\right)\right|^{2}+\ldots+2\left|\phi_{N+1}^{[1]}\left(\lambda_{2}\right)\right|^{2}},
\end{aligned}
$$

where $\phi_{d}^{[1]}\left(\lambda_{2}\right)=\left(\lambda_{2}-\lambda_{1}^{*}\right) \phi_{d}\left(\lambda_{2}\right)-\frac{C_{1}}{D_{1}}\left(\lambda_{1}-\lambda_{1}^{*}\right) \phi_{d}\left(\lambda_{1}\right)(d=1,2, \ldots, N+1)$ with

$$
C_{1}=\phi_{1}\left(\lambda_{2}\right) \phi_{1}^{*}\left(\lambda_{1}\right)+2 \phi_{2}\left(\lambda_{2}\right) \phi_{2}^{*}\left(\lambda_{1}\right)+\ldots 2 \phi_{N+1}\left(\lambda_{2}\right) \phi_{N+1}^{*}\left(\lambda_{1}\right)
$$




$$
D_{1}=\phi_{1}\left(\lambda_{1}\right) \phi_{1}^{*}\left(\lambda_{1}\right)+2 \phi_{2}\left(\lambda_{1}\right) \phi_{2}^{*}\left(\lambda_{1}\right)+\ldots 2 \phi_{N+1}\left(\lambda_{1}\right) \phi_{N+1}^{*}\left(\lambda_{1}\right) .
$$

$\phi_{1}^{[1]}\left(\lambda_{2}\right)$ needs to be a real function for DT (5) to be true, that's why we multiply $i$ in the expressions of $\phi_{1}\left(\lambda_{2}\right), \phi_{2}\left(\lambda_{2}\right), \ldots, \phi_{N+1}\left(\lambda_{2}\right)$ and let $\delta_{2 C}=\xi_{2 C}, \delta_{3 C}=\xi_{3 C}, \ldots, \delta_{N+1, C}=\xi_{N+1, C}$.

Via Variable Transformation (2), we can get the second-order soliton of System (1) as

$$
u_{j}^{[2]}(Z, T)=q_{j}^{[2]}(Z, T) e^{\frac{i}{6}\left(T-\frac{Z}{18}\right)} .
$$

\subsection{Evolution and interaction between the two bright solitons}

We find that there are $3 N+4$ real parameters $\eta_{1}, \eta_{2}, \delta_{1 R}, \xi_{1 R}, \delta_{h R}, \xi_{h R}$ and $\delta_{h C}(h=$ $2,3, \ldots, N+1)$ in Solutions (21). When these $3 N+4$ parameters take different values, the evolution and interaction (elastic or inelastic) between the two solitons also change accordingly.

We find that $\delta_{h C}$ do not affect the modulus value of $u_{j}^{[2]}$ in Solution (22). Then, we let $\delta_{h C}$ equal to 0 without affecting our following analysis.

(A) Elastic interaction case I:

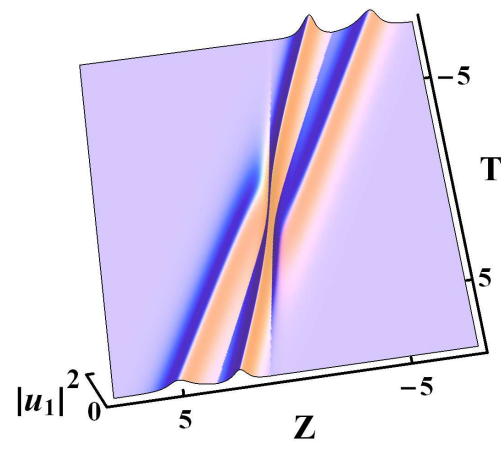

(a)

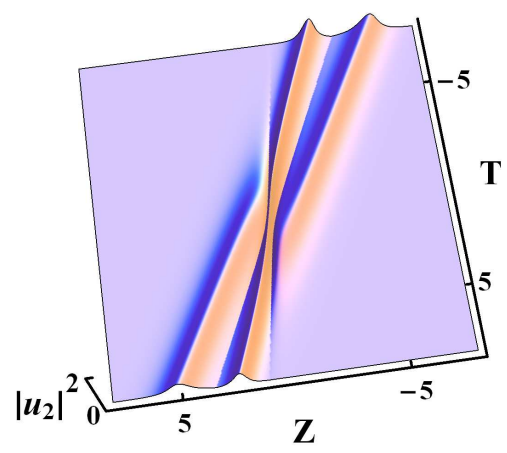

(b)

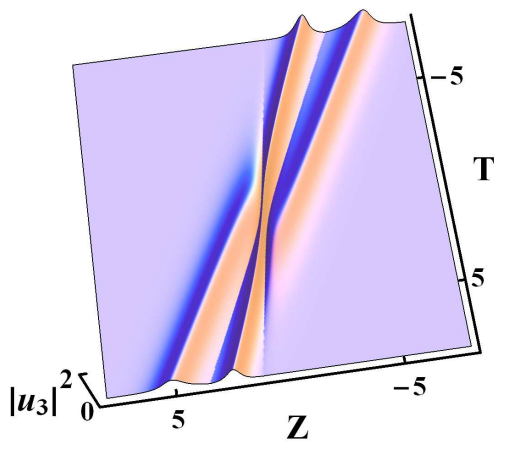

$(c)$

Figs. 2. Elastic interaction between the two solitons via Solutions (21) and (22) with $N=3, \eta_{1}=\frac{3}{4}, \eta_{2}=\frac{2}{3}, \delta_{1}=0, \delta_{2 R}=$ $0, \delta_{3 R}=0, \delta_{4 R}=0, \xi_{1}=0, \xi_{2 R}=0, \xi_{3 R}=0, \xi_{4 R}=0$.

Under the conditions: $\frac{\delta_{1}}{\xi_{1}}=\frac{\delta_{2 R}}{\xi_{2 R}}=\frac{\delta_{3 R}}{\xi_{4 R}}=\ldots=\frac{\delta_{N+1, R}}{\xi_{N+1, R}}=1$ or $\delta_{1}=\xi_{1}=\delta_{h R}=\xi_{h R}=0$, the interaction is elastic. It means that before and after interaction, the two solitons only have a phase shift. Their velocities, amplitudes, shapes, and widths do not change at all.

As shown in Figs. 2, the elastic interaction under the two conditions above occurs between the second-order solitons for System (1) with $N=3$.

The values of $\eta_{1}$ and $\eta_{2}$ affect the angle between the two solitons in elastic interaction. The angle increases as the value of ||$\eta_{1}|-| \eta_{2}||$ increases. The angle will reduce to zero but not equal to zero (means that the two solitons are nearly parallel showed in Figs. 5 when $\frac{\eta_{1}}{\eta_{2}} \sim-1$.

(B) Elastic interaction case II: 
Under the conditions: $-\delta_{1}=\delta_{h R}$ and $-\xi_{1}=\xi_{h R}$, the interaction is elastic and Solutions (22) reduce to

$$
u_{j}^{[2]}=\left(\eta_{2}^{2}-\eta_{1}^{2}\right) e^{\frac{i}{6}\left(T-\frac{Z}{18}\right)} \frac{2 \sqrt{2 N}\left(\eta_{2} \cosh \omega_{1}-\eta_{1} \cosh \omega_{2}\right)}{N\left(\eta_{1}-\eta_{2}\right)^{2} \cosh \left(\omega_{1}+\omega_{2}\right)+N\left(\eta_{1}+\eta_{2}\right)^{2} \cosh \left(\omega_{1}-\omega_{2}\right)-4 N \eta_{1} \eta_{2}},
$$

where

$$
\begin{aligned}
& \omega_{1}=-\left(8 \eta_{1}^{3}+\frac{1}{6} \eta_{1}\right) Z+2 \eta_{1} T+2 \delta_{1}-\frac{1}{2} \ln (2 N), \\
& \omega_{2}=-\left(8 \eta_{2}^{3}+\frac{1}{6} \eta_{2}\right) Z+2 \eta_{2} T+2 \xi_{1}-\frac{1}{2} \ln (2 N) .
\end{aligned}
$$

Motivated by Ref [26], without loss of generality, we assume that $0<\eta_{1}<\eta_{2}$. For Solution$\mathrm{s}(23)$, if $\omega_{1}$ is fixed, $\omega_{2}$ can be expressed as

$$
\omega_{2}=\frac{8 \eta_{2}^{3}+\frac{1}{6} \eta_{2}}{8 \eta_{1}^{3}+\frac{1}{6} \eta_{1}} \omega_{1}+\frac{16 \eta_{2}\left(\eta_{1}^{2}-\eta_{2}^{2}\right)}{8 \eta_{1}^{2}+\frac{1}{6}} T+2 \xi_{1}-\frac{1}{2} \ln (2 N)-\frac{8 \eta_{2}^{3}+\frac{1}{6} \eta_{2}}{8 \eta_{1}^{3}+\frac{1}{6} \eta_{1}}\left[2 \delta_{1}-\frac{1}{2} \ln (2 N)\right] .
$$

When $T \rightarrow-\infty$, we have $e^{-\omega_{2}} \rightarrow 0$; When $T \rightarrow+\infty$, we have $e^{\omega_{2}} \rightarrow 0$. We can get

$$
\lim _{\substack{T \rightarrow-\infty \\ \omega_{1} \text { fixed }}} u_{j}^{[2]}=\frac{-2 \eta_{1}}{\sqrt{2 N}} e^{\frac{i}{6}\left(T-\frac{Z}{18}\right)} \operatorname{sech}\left[\omega_{1}+\ln \left(\frac{\eta_{2}-\eta_{1}}{\eta_{2}+\eta_{1}}\right)\right]
$$

and

$$
\lim _{\substack{t \rightarrow+\infty \\ \omega_{1} \text { fixed }}} u_{j}^{[2]}=\frac{-2 \eta_{1}}{\sqrt{2 N}} e^{\frac{i}{6}\left(T-\frac{Z}{18}\right)} \operatorname{sech}\left[\omega_{1}+\ln \left(\frac{\eta_{2}+\eta_{1}}{\eta_{2}-\eta_{1}}\right)\right] .
$$

If $\omega_{2}$ is fixed, $\omega_{1}$ can be expressed as

$$
\omega_{1}=\frac{8 \eta_{1}^{3}+\frac{1}{6} \eta_{1}}{8 \eta_{2}^{3}+\frac{1}{6} \eta_{2}} \omega_{2}+\frac{16 \eta_{1}\left(\eta_{2}^{2}-\eta_{1}^{2}\right)}{8 \eta_{2}^{2}+\frac{1}{6}} T+2 \xi_{1}-\frac{1}{2} \ln (2 N)-\frac{8 \eta_{1}^{3}+\frac{1}{6} \eta_{1}}{8 \eta_{2}^{3}+\frac{1}{6} \eta_{2}}\left[2 \delta_{1}-\frac{1}{2} \ln (2 N)\right] .
$$

When $T \rightarrow-\infty$, we have $e^{\omega_{1}} \rightarrow 0$; When $T \rightarrow+\infty$, we have $e^{-\omega_{1}} \rightarrow 0$. So we can get

$$
\lim _{\substack{T \rightarrow-\infty \\ \omega_{2} \text { fixed }}} u_{j}^{[2]}=\frac{-2 \eta_{2}}{\sqrt{2 N}} e^{\frac{i}{6}\left(T-\frac{Z}{18}\right)} \operatorname{sech}\left[\omega_{2}+\ln \left(\frac{\eta_{2}+\eta_{1}}{\eta_{2}-\eta_{1}}\right)\right],
$$

and

$$
\lim _{\substack{T \rightarrow+\infty \\ \omega_{2} \text { fixed }}} u_{j}^{[2]}=\frac{-2 \eta_{2}}{\sqrt{2 N}} e^{\frac{i}{6}\left(T-\frac{Z}{18}\right)} \operatorname{sech}\left[\omega_{2}+\ln \left(\frac{\eta_{2}-\eta_{1}}{\eta_{2}+\eta_{1}}\right)\right] .
$$

All the two solitons own the same shape, amplitude and velocity. Results of the asymptotic analysis show that after the elastic interaction, the properties changed are merely the characteristic lines.

(C) Inelastic interaction:

Under the conditions: $\frac{\delta_{1}}{\xi_{1}}=\frac{\delta_{2 R}}{\xi_{2 R}}=\frac{\delta_{3 R}}{\xi_{4 R}}=\ldots=\frac{\delta_{N+1, R}}{\xi_{N+1, R}} \neq 0,1$, the interaction is inelastic. We take the second-order soliton for System (1) with $N=3$ as an example, inelastic interaction occurs as seen in Figs. 3 and 4 . When $k \gg 1$, the solitons $S 1$ in the $u_{1}, u_{2}$ components are 
suppressed and the soliton $S 1$ in the $u_{3}$ component is enhanced; While the solitons $S 2$ in the $u_{1}, u_{2}$ components are enhanced and the soliton $S 2$ in the $u_{3}$ component is suppressed.

When $k \ll-1$, the soliton $S 1$ in the $u_{1}$ component keeps unchanged and the solitons $S 1$ in the $u_{2}, u_{3}$ components are suppressed; While the solitons $S 2$ in the $u_{2}, u_{3}$ components keep unchanged and the soliton $S 2$ in the $u_{1}$ component is suppressed.

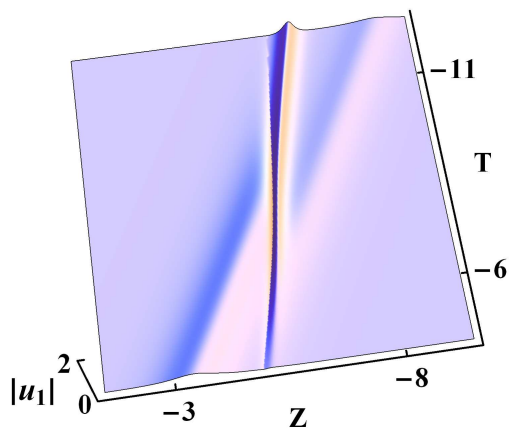

$(a)$

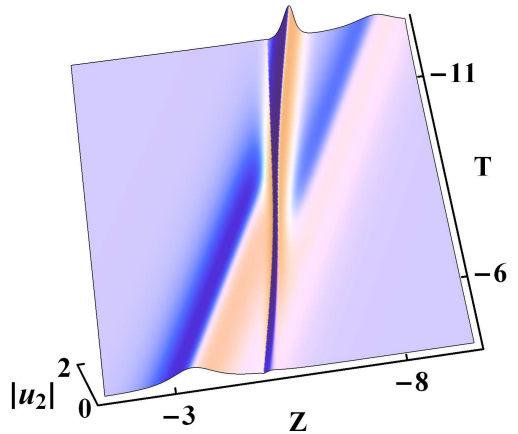

$(b)$

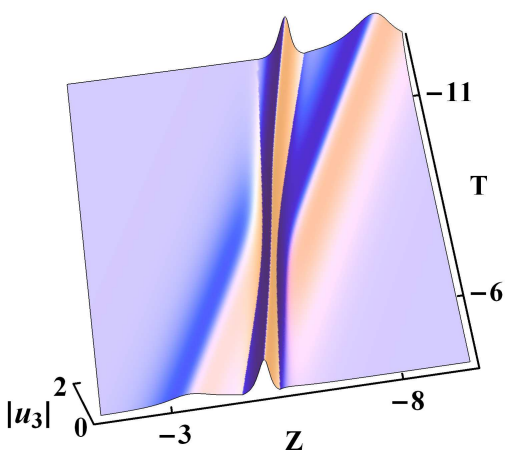

(c)

Figs. 3. Inelastic interaction between the two solitons via Solutions (21) and (22) with $N=3, \eta_{1}=\frac{2}{3}, \eta_{2}=1, \delta_{1}=1, \delta_{2 R}=$ $2, \delta_{3 R}=3, \delta_{4 R}=4, \xi_{1}=10, \xi_{2 R}=20, \xi_{3 R}=30$ and $\xi_{4 R}=40$.

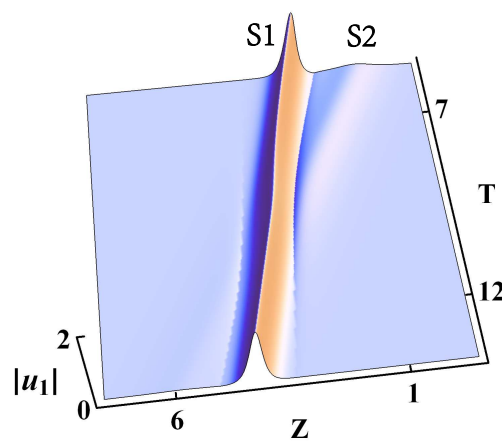

(a)

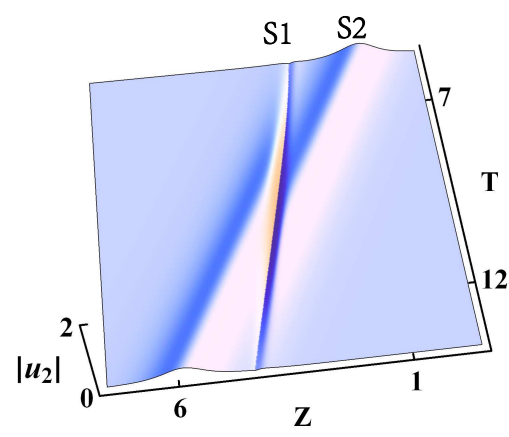

(b)

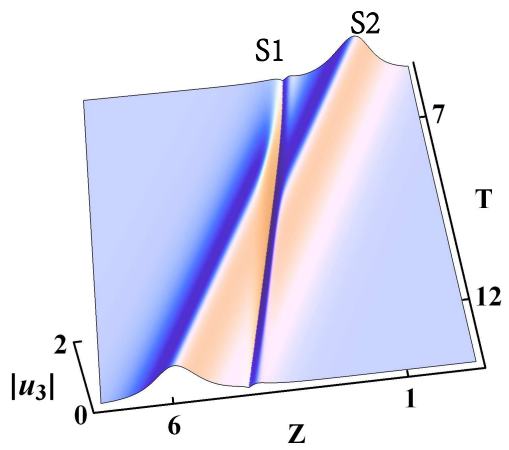

(c)

Figs. 4. The same as Figs. 3 except that $\xi_{1}=-10, \xi_{2 R}=-20, \quad \xi_{3 R}=-30$ and $\xi_{4 R}=-40$.

(D) Nearly parallel:

Under the conditions: $\frac{\delta_{1}}{\xi_{1}}=\frac{\delta_{2 R}}{\xi_{2 R}}=\frac{\delta_{3 R}}{\xi_{4 R}}=\ldots=\frac{\delta_{N+1, R}}{\xi_{N+1, R}}=p$ (constant) $\neq 0$ and $\frac{\eta_{1}}{\eta_{2}} \sim-1$, the two solitons are nearly parallel. However, the two solitons won't be parallel because the second-order soliton solutions become zero when $\eta_{1}+\eta_{2}=0$. As seen in Figs. 5, the characteristic lines of two solitons for System (3) with $N=3$ are nearly parallel. As long as $\left|\eta_{1}\right| \neq\left|\eta_{2}\right|$, the two solitons will eventually meet and collide elastically or inelastically. 


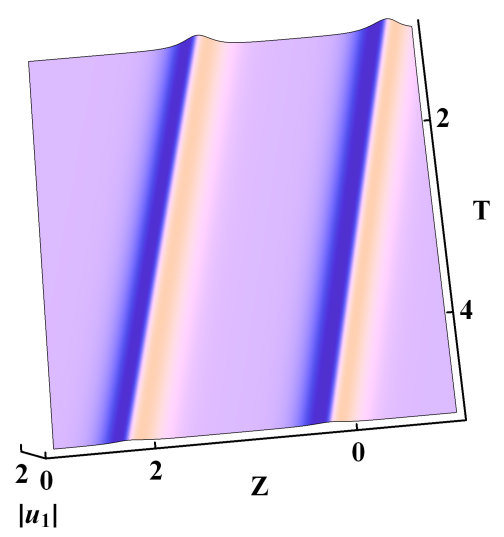

(a)

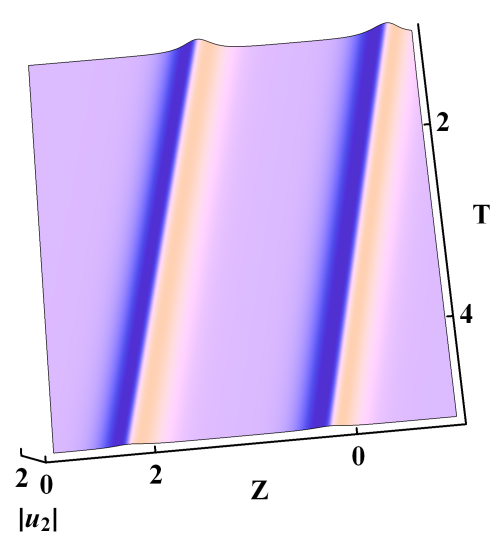

(b)

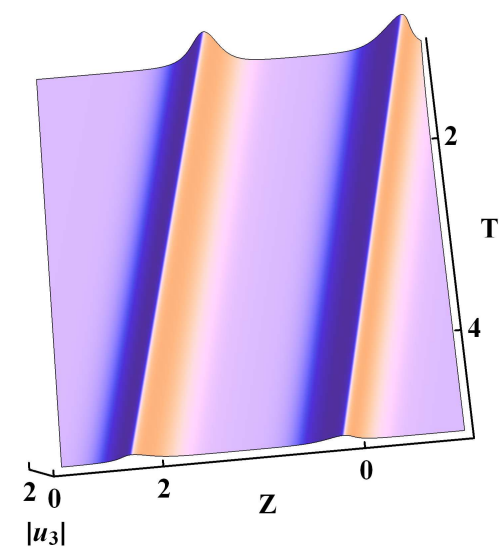

$(c)$

Figs. 5. Nearly parallel two solitons via Solutions (21) and (22) with $N=3, \eta_{1}=1, \eta_{2}=-0.99, \delta_{1}=2, \delta_{2 R}=3, \delta_{3 R}=3, \delta_{4 R}=$ $4, \xi_{1}=2, \xi_{2 R}=3, \xi_{3 R}=3$ and $\xi_{4 R}=4$.

\section{Bright and dark-bright solitons of System (1) on the plane wave background}

To get the bright and dark-bright solitons, we choose the following plane wave seed solutions of System (3):

$$
q_{j}(x, t)=a_{j} e^{i \theta_{j}(x, t)}, \theta_{j}(x, t)=b_{j} x+c_{j} t, c_{j}=b_{j}^{3}-6 b_{j} \sum_{g=1}^{N} a_{g}^{2},
$$

where $a_{j}$ and $b_{j}$ are real constants, $b_{j}$ are all distinct, $g$ is a positive integer, $a_{j} \geq 0$.

According to Solutions (14), we firstly need to solve Lax Pair (4) with Seed Solutions (24) via the gauge transformation:

$$
\Phi=D \Psi, D=\operatorname{diag}\left(1, e^{-i \theta_{1}}, e^{i \theta_{1}}, e^{-i \theta_{2}}, e^{i \theta_{2}}, \ldots, e^{-i \theta_{N}}, e^{i \theta_{N}}\right) .
$$

$\Psi$ satisfies the following Lax pair:

$$
\begin{gathered}
\Psi_{x}=K \Psi \\
\Psi_{t}=\left[-K^{3}+3 i \lambda K^{2}+\left(3 \lambda^{2}-6 \sum_{g=1}^{N} a_{g}^{2}\right) K+3 i \lambda\left(\lambda^{2}+2 \sum_{g=1}^{N} a_{g}^{2}\right)\right] \Psi,
\end{gathered}
$$

where

$$
K=D^{-1}\left(U D-D_{x}\right)=\left(\begin{array}{cccccc}
-i \lambda & a_{1} & a_{1} & \ldots & a_{N} & a_{N} \\
-a_{1} & i\left(\lambda+b_{1}\right) & 0 & \ldots & 0 & 0 \\
-a_{1} & 0 & i\left(\lambda-b_{1}\right) & \ldots & 0 & 0 \\
\vdots & \vdots & \vdots & \ddots & \vdots & \vdots \\
-a_{N} & 0 & 0 & \ldots & i\left(\lambda+b_{N}\right) & 0 \\
-a_{N} & 0 & 0 & \ldots & 0 & i\left(\lambda-b_{N}\right)
\end{array}\right)
$$


In order to convert $K$ to a diagonal matrix, we proceed the following matrix decomposition

$$
K M=M K_{0}, K_{0}=\operatorname{diag}\left(\mu_{1}+i \lambda, \mu_{2}+i \lambda, \mu_{3}+i \lambda, \ldots, \mu_{2 N+1}+i \lambda\right),
$$

where

$$
M=\left(\begin{array}{cccc}
1 & 1 & \cdots & 1 \\
\frac{-a_{1}}{\mu_{1}-i b_{1}} & \frac{-a_{1}}{\mu_{2}-i b_{1}} & \cdots & \frac{-a_{1}}{\mu_{2 N+1}-i b_{1}} \\
\frac{-a_{1}}{\mu_{1}+i b_{1}} & \frac{-a_{1}}{\mu_{2}+i b_{1}} & \cdots & \frac{-a_{1}}{\mu_{2 N+1}+i b_{1}} \\
\frac{-a_{2}}{\mu_{1}-i b_{2}} & \frac{-a_{2}}{\mu_{2}-i b_{2}} & \cdots & \frac{-a_{2}}{\mu_{2 N+1}-i b_{2}} \\
\frac{-a_{2}}{\mu_{1}+i b_{2}} & \frac{-a_{2}}{\mu_{2}+i b_{2}} & \cdots & \frac{-a_{2}}{\mu_{2 N+1}+i b_{2}} \\
\cdots & \ldots & \cdots & \ldots \\
\frac{-a_{N}}{\mu_{1}-i b_{N}} & \frac{-a_{N}}{\mu_{2}-i b_{N}} & \cdots & \frac{-a_{N}}{\mu_{2 N+1}-i b_{N}} \\
\frac{-a_{N}}{\mu_{1}+i b_{N}} & \frac{-a_{N}}{\mu_{2}+i b_{N}} & \cdots & \frac{-a_{N}}{\mu_{2 N+1}+i b_{N}}
\end{array}\right) .
$$

$\mu_{1}, \mu_{2}, \ldots, \mu_{2 N+1}$ are $2 N+1$ distinct roots of the $(2 N+1)$ th-order characteristic equation in the variable $\mu$ :

$$
\operatorname{det}\left[(\mu+i \lambda) I_{2 N+1}-K\right]=0,
$$

where det denotes the determinant of a matrix.

We simplify the $(2 N+1)$ th-order characteristic equation, then get

$$
\mu+2 i \lambda+\frac{a_{1}^{2}}{\mu+i b_{1}}+\frac{a_{1}^{2}}{\mu-i b_{1}}+\frac{a_{2}^{2}}{\mu+i b_{2}}+\frac{a_{2}^{2}}{\mu-i b_{2}}+\ldots+\frac{a_{N}^{2}}{\mu+i b_{N}}+\frac{a_{N}^{2}}{\mu-i b_{N}}=0 .
$$

Eigenfunctions (25) can be given as $\Phi=D M Q L$ with

$$
\begin{aligned}
Q & =\operatorname{diag}\left(e^{F\left(\mu_{1}\right)}, e^{F\left(\mu_{2}\right)}, \ldots, e^{F\left(\mu_{2 N+1}\right)}\right), \\
F(\mu) & =(\mu+i \lambda) x+\left[-(\mu+i \lambda)^{3}+3 i \lambda(\mu+i \lambda)^{2}+\left(3 \lambda^{2}-6 \sum_{g=1}^{N} a_{g}^{2}\right)(\mu+i \lambda)\right] t \\
& =A(x, t)+B(\mu), \\
A(x, t) & =i \lambda x+4 i \lambda^{3} t, B(\mu)=\mu\left(x-6 \sum_{j=1}^{N}\left(a_{j}^{2}\right) t-\mu^{2} t\right), \\
L & =\left(l_{1}, l_{2}, \ldots, l_{2 N+1}\right)^{T},
\end{aligned}
$$

where $l_{m}(m=1,2, \ldots, 2 N+1)$ are non-zero real constants.

After calculation we get:

$$
\Phi=D M Q L=\left(\begin{array}{c}
\phi_{1} \\
\phi_{2} \\
\phi_{2}^{*} \\
\cdots \\
\phi_{N} \\
\phi_{N}^{*}
\end{array}\right)=\left(\begin{array}{c}
e^{A(x, t)} \sum_{m=1}^{2 N+1} l_{m} e^{B\left(\mu_{m}\right)} \\
e^{A(x, t)} a_{1} e^{-\theta_{1}} \sum_{m=1}^{2 N+1} \frac{l_{m} e^{B\left(\mu_{m}\right)}}{i b_{1}-\mu_{m}} \\
e^{A(x, t)} a_{1} e^{\theta_{1}} \sum_{m=1}^{2 N+1} \frac{l_{m} e^{B\left(\mu_{m}\right)}}{-i b_{1}-\mu_{m}} \\
\ldots \\
e^{A(x, t)} a_{N} e^{-\theta_{N}} \sum_{m=1}^{2 N+1} \frac{l_{m} e^{B\left(\mu_{m}\right)}}{i b_{N}-\mu_{m}} \\
e^{A(x, t)} a_{N} e^{\theta_{N}} \sum_{m=1}^{2 N+1} \frac{l_{m} e^{B\left(\mu_{m}\right)}}{-i b_{N}-\mu_{m}}
\end{array}\right) .
$$


To make sure that $\phi_{1}$ is a real function and $\Phi$ satisfies DT (5), $l_{m}$ and $\mu_{m}$ need be real while $\lambda$ need be a pure imaginary. It also means that all the solutions of the $(2 N+1)$-order Characteristic Equation (26) need be real.

\subsection{The first-order bright soliton of System (1)}

Without loss of generality, we suppose that $\lambda=i \xi, \mu_{1}, \mu_{2}>0$ and $\mu_{1} \neq \mu_{2}$. Under this constraints $l_{3}=l_{4}=\ldots=l_{2 N+1}=0$, we substitute Eq. (27) into Solutions (16) and use the Variable Transformation (2), then we get the first-order bright soliton

$$
\begin{aligned}
& u_{j}^{[1]}=a_{j} e^{i\left[\left(b_{j}+\frac{1}{6}\right) T+\left(c_{j}-\frac{b_{j}}{12}-\frac{1}{108}\right) Z\right]}\left(1-\frac{G_{1}+G_{2} \cosh \left[B\left(\mu_{1}\right)-B\left(\mu_{2}\right)+G_{3}\right]}{H_{1}+H_{2} \cosh \left[B\left(\mu_{1}\right)-B\left(\mu_{2}\right)+H_{3}\right]}\right), \\
& G_{1}=\frac{l_{1} l_{2}}{i b_{j}+\mu_{1}}+\frac{l_{1} l_{2}}{i b_{j}+\mu_{2}}, G_{2}=\frac{2 l_{2}}{\sqrt{\left(i b_{j}+\mu_{1}\right)\left(i b_{j}+\mu_{2}\right)}}, \\
& G_{3}=\frac{1}{2} \ln \frac{l_{1}^{2}\left(i b_{j}+\mu_{2}\right)}{l_{2}^{2}\left(i b_{j}+\mu_{1}\right)}, H_{1}=\frac{2 l_{1} l_{2}}{\mu_{1}+\mu_{2}}, \\
& H_{2}=\frac{l_{1} l_{2}}{\sqrt{\mu_{1} \mu_{2}}}, H_{3}=\frac{1}{2} \ln \frac{l_{1}^{2} \mu_{2}}{l_{2}^{2} \mu_{1}} . \\
& B(\mu)=\mu\left(T-6 \sum_{j=1}^{N}\left(a_{j}^{2}\right) Z-\mu^{2} Z-\frac{Z}{12}\right) .
\end{aligned}
$$

We find that the breathers don't appear under this condition. $q_{j}$ are always a series of bright solitons whether $l_{m}$ are equal to zero or not.

We can obtain the following properties of the one soliton:

(a) Soliton Velocities: $V\left(u_{j}^{[1]}\right)=\frac{1}{\mu_{1}^{2}+\mu_{2}^{2}+\mu_{1} \mu_{2}+6 \sum_{j=1}^{N} a_{j}^{2}+\frac{1}{12}}$,

(b) Soliton Widths: $W\left(u_{j}^{[1]}\right)=\frac{1}{\mu_{1}-\mu_{2}}$.

We take the first-order bright soliton for System (3) with $N=1$ as examples. In order for the three solutions $\left(\mu_{1}, \mu_{2}, \mu_{3}\right)$ of the cubic equation to be real, discriminant involving three parameters $\left(a_{1}, b_{1}, \xi\right)$ need to satisfy

$$
\frac{1}{27}\left(2 a_{1}^{2}+b_{1}^{2}-\frac{4 \xi^{2}}{3}\right)^{3}+\frac{4}{9} \xi^{2}\left(a_{1}^{2}-b_{1}^{2}-\frac{4 \xi^{2}}{9}\right)^{2} \leq 0 .
$$

When $N>1$, the Characteristic Equation (26) is at least fifth degree, which means that there is no root formula.

\subsection{The first-order dark-bright soliton of System (1)}

In order to get the dark-bright soliton of System (1), without loss of generality, motivated by Ref [27], we assume that there are $N_{1}$ dark solitons $\left(N_{1}\right.$ is a positive integer and $\left.1 \leq N_{1}<N\right)$ and $N-N_{1}$ bright solitons. For this we let $a_{f}=0\left(f=1,2, \ldots, N_{1}\right)$, means that $u_{f}(x, t)$ are dark solitons and the rest $\left(N-N_{1}\right)$ components $u_{e}(x, t)\left(e=N_{1}+1, N_{1}+2, \ldots, N\right)$ are bright solitons. 
In order to make Solitons (14) hold, we let $\lambda=i \xi, l_{2}=l_{3}=l_{3}=\ldots=l_{2 N_{1}+1}=0$ and $l_{2 e}=l_{2 e+1}$. Suppose that $\xi \mu_{1}>0$, then the first-order dark-bright soliton of System (1) can be derived as

$$
\begin{aligned}
& u_{f}^{[1]}=a_{f} e^{i\left[\left(b_{f}+\frac{1}{6}\right) T+\left(c_{f}-\frac{b_{f}}{12}-\frac{1}{108}\right) Z\right]}\left\{1-\frac{\mu_{1}}{i b_{f}+\mu_{1}}\left[1+\tanh \left(B\left(\mu_{1}\right)+E_{1}\right)\right]\right\}, \\
& E_{1}=\frac{1}{2} \ln \frac{l_{1}^{2} \xi}{\mu_{1} \sum_{e=N_{1}+1}^{N} l_{2 e+1}^{2}}, \\
& u_{e}^{[1]}=\sqrt{\xi \mu_{1}} \operatorname{sech}\left[B\left(\mu_{1}\right)+E_{2}\right], \\
& E_{2}=\frac{1}{2} \ln \frac{l_{1}^{2} \xi}{\mu_{1} l_{2 e+1}^{2}}, \\
& B\left(\mu_{1}\right)=\mu_{1}\left(T-6 \sum_{j=1}^{N}\left(a_{j}^{2}\right) Z-\mu_{1}^{2} Z-\frac{Z}{12}\right) .
\end{aligned}
$$

When $x \rightarrow-\infty, u_{f}^{[1]} \rightarrow u_{f}$ and $u_{e}^{[1]} \rightarrow 0$. When $x \rightarrow+\infty, u_{f}^{[1]} \rightarrow i b_{f} /\left(i b_{f}+\mu_{1}\right) u_{f}$ and $u_{e}^{[1]} \rightarrow 0$. The results of asymptotic analysis show that $u_{f}^{[1]}$ approach to constant amplitudes $\left|u_{f}\right|$ and $\left|b_{f} / \sqrt{b_{f}^{2}+\mu_{1}^{2}}\right|\left|u_{f}\right|$ as $x \rightarrow \pm \infty$, respectively. $u_{e}^{[1]}$ approach to zero both as $x \rightarrow \pm \infty$.

We can obtain the following properties:

(a) Soliton Velocities: $V\left(u_{f}^{[1]}\right)=V\left(u_{e}^{[1]}\right)=6 \sum_{j=1}^{N_{1}}\left(a_{j}^{2}\right)+\mu_{1}^{2}+\frac{Z}{12}$,

(b) Soliton Amplitudes: $A\left(u_{f}^{[1]}\right)=\frac{\left|\mu_{1}\right|}{i b_{j}+\mu_{1}}, A\left(u_{e}^{[1]}\right)=\sqrt{\xi \mu_{1}}$,

(c) Soliton Widths: $W\left(u_{f}^{[1]}\right)=W\left(u_{e}^{[1]}\right)=\frac{1}{\mu_{1}}$,

(d) Total Soliton Energy: $E=\int_{-\infty}^{+\infty}\left(\left.u_{1}^{[1]}\right|^{2}+\ldots+\left|u_{N}^{[1]}\right|^{2}\right) d x=\sum_{f=1}^{N_{1}} \frac{\mu_{1}^{2}}{\left(i b_{f}+\mu_{1}\right)^{2}}+\left(N-N_{1}\right) \xi \mu_{1}$,

(e) Characteristic Lines: $\mu_{1}\left(T-6 \sum_{j=1}^{N}\left(a_{j}^{2}\right) Z-\mu_{1}^{2} Z-\frac{Z}{12}\right)+E_{1}=0$ for $u_{f}^{[1]}$ and $\mu_{1}(T-$ $\left.6 \sum_{j=1}^{N}\left(a_{j}^{2}\right) Z-\mu_{1}^{2} Z-\frac{Z}{12}\right)+E_{2}=0$ for $u_{e}^{[1]}$.

All the dark solitons $u_{f}^{[1]}$ and bright solitons $u_{e}^{[1]}$ move at the same velocity. We find that $\left|b_{j} u_{j} / \sqrt{b_{j}^{2}+\mu_{1}^{2}}\right|^{2}<\left|u_{j}\right|^{2}$ and $\sqrt{\xi \mu_{1}}>0$, which imply that the amplitudes of $u_{f}^{[1]}$ is lower than the background, but the amplitude of $u_{e}^{[1]}$ is higher than the background. This also verified the previous $N_{1}$ components $u_{f}^{[1]}$ are dark solitons and the rest $N-N_{1}$ components $u_{e}^{[1]}$ are bright solitons.

To illustrate these dark-bright solitons, we choose the first-order dark-dark-bright solitons for System (1) with $N=3$ as an example as showed in Figs. 6 (corresponding to three identical characteristic roots). 


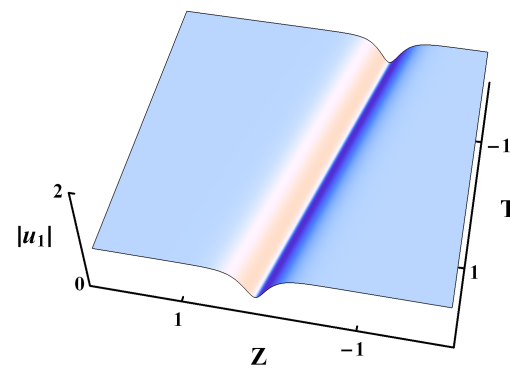

$(a)$

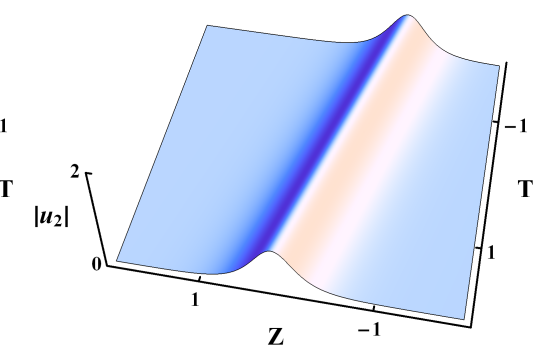

(b)

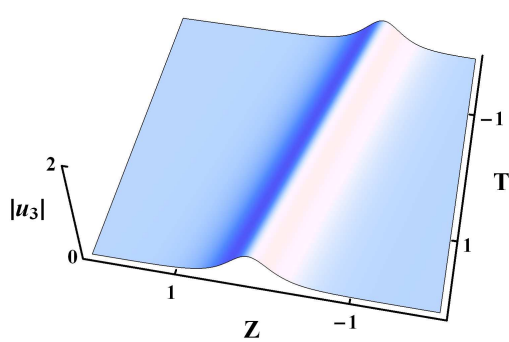

$(c)$

Figs. 6. Dark-bright-bright soliton via Solutions (29) with $N=3, a_{1}=\frac{\sqrt{5}}{3}, b_{1}=\frac{1}{3}, a_{2}=a_{3}=0, \xi=1, \mu_{1}=1, \mu_{2}=\frac{1}{3}, \mu_{3}=$ $\frac{2}{3}, l_{1}=1, l_{2}=l_{3}=0, l_{5}=3$ and $l_{7}=2$.

\section{Conclusion}

In this paper, an $N$-coupled HNLS system ( $N$ is a positive integer), i.e., System (1) which describes the properties of the ultrashort optical pulses in an optical fiber, has been investigated. System (1) has been converted into System (3) through Variable Transformation (2). Based on the $(2 N+1)$ th-order Lax Pair (4) of System (3), DT (5) for System (1) has been constructed, which demands that the first element $\phi_{1}$ in the eigenfunction $\Phi$ is real and complex spectral parameter $\lambda_{1}=i \eta_{1}$ is a pure imaginary number. By virtue of DT (5), the $n$ th-iterated solutions of System (1) ( $n$ is a positive integer) have been constructed as Solution (16).

Analytic forms of the first-order soliton on the zero background have been got as Solutions (19). Properties of the solitons have been investigated: Solitons enjoy the same velocity, width and characteristic line; Widths of the solitons are inverse to $\eta_{1}$ while their velocities decrease as $\eta_{1}$ increase; Velocities of the solitons are always positive; Amplitudes of the solitons in each component are proportional to $e^{\delta_{j+1, R}} \quad\left(\delta_{j+1, R}\right.$ are the integral constants), as is shown in Figs. 1.

Analytic forms of the second-order soliton on the zero background have been got as Solutions (21) and (22) with $3 N+4$ real parameters. Effects of those parameters on the two solitons have been analysed by virtue of the asymptotic analysis:

- Elastic interaction case I and case II have been shown in Figs. 2. Via the asymptotic analysis, we have found that before and after the interaction, the two solitons only have a phase shift.

- Inelastic interaction between the two solitons have been shown in Figs. 3 and 4. We have analyzed the amplitude changes of solitons $S 1$ and $S 2$ in each component, which imply the increase or decrease of soliton energy.

- Nearly parallel two solitons have been shown in Figs. 5. As long as the spectral parameters $\lambda_{1} \neq-\lambda_{2}$, the two solitons will eventually meet and interact elastically or inelastically. 
The first-order bright and dark-bright soliton for System (1) has been derived on the plane wave background as Solutions (28) and (29), respectively. Properties of these solitons have been investigated: Velocities and widths of bright solitons in Solutions (28) are related to the two solutions $\left(\mu_{1}\right.$ and $\left.\mu_{2}\right)$ of (26); All the dark solitons and bright solitons in Solutions (29) move at the same velocity as shown in Figs. 6.

\section{Acknowledgement}

We sincerely thank all members of the discussion group for their valuable comments. This work has been supported by the National Natural Science Foundation of China under Grant No. 11772017, and by the Fundamental Research Funds for the Central Universities.

\section{Ethics declarations}

\section{Conflicts of interest}

The authors declare that they have no conflict of interest.

\section{Data availability}

Data sharing not applicable to this article as no datasets were generated or analysed during the current study.

\section{References}

[1] Yuan, Y.Q., Tian, B., Qu, Q.X., et al.: Lax pair, binary Darboux transformation and dark solitons for the three-component Gross-Pitaevskii system in the spinor Bose-Einstein condensate. Nonlinear Dyn. 99(4), 3001-3011 (2020).

[2] Ji, T., Zhai, Y.: Soliton, breather and rogue wave solutions of the coupled Gerdjikov-Ivanov equation via Darboux transformation. Nonlinear Dyn. 101(1), 619-631 (2020).

[3] Amjad, Z., Haider, B.: Binary Darboux transformation of time-discrete generalized lattice Heisenberg magnet model. Chaos Soliton. Fract. 130, 109404 (2020).

[4] Maddouri, K., Azzouzi, F., Triki, H., et al.: Dark-managed solitons in inhomogeneous cubicquintic-septimal nonlinear media. Nonlinear Dyn. 103(3), 2793-2803 (2021).

[5] Lü, X., Lin, F.: Soliton excitations and shape-changing collisions in alpha helical proteins with interspine coupling at higher order. Commun. Nonlinear Sci. Numer. Simulat. 32, 241261 (2016). 
[6] Zhao, X., Tian, B., Tian, H.Y., et al.: Bilinear Bäcklund transformation, Lax pair and interactions of nonlinear waves for a generalized $(2+1)$-dimensional nonlinear wave equation in nonlinear optics/fluid mechanics/plasma physics. Nonlinear Dyn. 103(2), 1785-1794 (2021).

[7] Hanif, Y., Saleem, U.: Darboux transformation and multi-soliton solutions of the discrete sine-Gordon equation. Prog. Theor. Exp. Phys. 2020(6), 063A01 (2020).

[8] Wang, X.B., Han, B.: The three-component coupled nonlinear Schrödinger equation: Rogue waves on a multi-soliton background and dynamics. Europhys. Lett. 126(1), 15001 (2019).

[9] Pal, R., Kaur, H., Raju, T.S., et al.: Periodic and rational solutions of variable-coefficient modified Korteweg-de Vries equation. Nonlinear Dyn. 89(1), 617-622 (2017).

[10] Nandy, S., Sarma, D., Barthakur A., Zhang, A.: Hirota bilinearization of coupled higherorder nonlinear Schrödinger equation. Commun. Nonlinear Sci. Numer. Simulat. 15(11), 3386-3389 (2010).

[11] Nakkeeran, K.: Bright and dark optical solitons in fiber media with higher-order effects. Chaos Soliton. Fract. 13(4), 673-679 (2002).

[12] Nandy, S.: Inverse scattering approach to coupled higher-order nonlinear Schrödinger equation and N-soliton solutions. Nucl. Phys. B 679(3), 647-659 (2004).

[13] Lü, X., Li, J., Zhang, H.Q., Xu T., Li, L.L.: Integrability aspects with optical solitons of a generalized variable-coefficient N-coupled higher order nonlinear Schrödinger system from inhomogeneous optical fibers. J. Math. Phys. 51(4), 043511 (2010).

[14] Guo, B.L., Wang, Y.F.: Mixed-type soliton solutions for the N-coupled higher-order nonlinear schrödinger equation in optical fibers. Chaos Soliton. Fract. 93, 246-251 (2016).

[15] Nakkeeran, K., Porsezian, K., Shanmugha, P.S., Mahalingam, A.: Optical solitons in Ncoupled higher order nonlinear Schrödinger equations. Phys. Rev. Lett. 80(7), 1425 (1998).

[16] Sasa, N., Satsuma, J.: New-type of soliton solutions for a higher-order nonlinear Schrödinger equation. J. Phys. Soc. Jpn. 60(2), 409-417 (1991).

[17] Yang, J.J., Tian, S.F., Peng W.Q., Borah, T.T.: The N-coupled higher-order nonlinear Schrödinger equation: Riemann-Hilbert problem and multi-soliton solutions. Math. Meth. Appl. Sci. 43(5), 2458-2472 (2020).

[18] Seenuvasakumaran, P., Mahalingam, A., Porsezian, K.: Dark solitons in N-coupled higher order nonlinear Schrödinger equations. Commun. Nonlinear Sci. Numer. Simulat. 13(7), 1318-1328 (2008). 
[19] Liu, Y., Gao, Y.T., Xu, T., et al.: Soliton solution, Bäcklund transformation, and conservation laws for the Sasa-Satsuma equation in the optical fiber communications. Z. Naturforsch. A 65(4), 291-300 (2014).

[20] Wright III, O.C.: Sasa-Satsuma equation, unstable plane waves and heteroclinic connections. Chaos Soliton. Fract. 33(2), 374-387 (2007).

[21] Bindu, S.G., Mahalingam, A., Porsezian, K.: Dark soliton solutions of the coupled Hirota equation in nonlinear fiber. Phys. Lett. A 286(5), 321-331 (2001).

[22] Lü, X.: Bright-soliton collisions with shape change by intensity redistribution for the coupled Sasa-Satsuma system in the optical fiber communications. Commun. Nonlinear Sci. Numer. Simul. 19(11), 3969-3987 (2014).

[23] Chen, S.S, Tian, B., Liu, L., et al.: Conservation laws, binary Darboux transformations and solitons for a higher-order nonlinear Schrödinger system. Chaos Soliton. Fract. 118, 337-346 (2019).

[24] Porsezian, K., Sundaram, P.S., Mahalingam, A.: Complete integrability of N-coupled higherorder nonlinear Schrödinger equations in nonlinear optics. J. Phys. A-Math. Gen. 32(49), 8731 (1999).

[25] Matveev, V.B., Salle, M.A.: Darboux Transformations and Solitons. Springer, Berlin(1991).

[26] Yu, X., Gao, Y.T., Sun, Z.Y., et al.: N-soliton solutions for the (2+1)-dimensional HirotaMaccari equation in fluids, plasmas and optical fibers. J. Math. Anal. Appl. 378(2), 519-527 (2011).

[27] Zhang, G., Yan, Z.: The n-component nonlinear Schrödinger equations: dark-bright mixed N- and high-order solitons and breathers, and dynamics. Proc. R. Soc. A-Math. Phys. Eng. Sci. 474(2215), 20170688 (2018). 